\title{
On the convergence of iterative discrete bitloading for autonomous spectrum management in DSL systems
}

\author{
Rafael Ferrari, Renato R. Lopes and João M. T. Romano
}

\begin{abstract}
Resumo-Este trabalho analisa o impacto da restrição de alocações discretas de bits na convergência de um algoritmo iterativo para gerenciamento espectral distribuído em sistemas DSL. O problema de controle de potência é formulado como um jogo não-cooperativo no qual os usuários otimizam unilateralmente suas alocações de bits e de potência de transmissão de modo a maximizar a taxa. Neste contexto é mostrado que, dependendo das características do canal de comunicação, a inclusão da restrição de alocação discreta de bits no problema de otimização pode levar a jogos com múltiplos pontos de equilíbrio de Nash ou mesmo a jogos onde não há nenhum ponto de equilíbrio. Simulações em cenários DSL representativos são apresentadas de modo a comparar o algoritmo com restrição discreta de alocação de bits com o bem conhecido algoritmo iterative water-filling.
\end{abstract}

Palavras-Chave-Digital subscriber lines, Gerenciamento espectral distribuído, Sistemas multiportadora.

Abstract-This work analyzes the impact of the discrete bitloading constraint on the convergence of a iterative algorithm for distributed spectrum management in a DSL environment. The power control problem is formulated as a noncooperative game in which the users unilaterally optimize their transmit power and bit allocations in order to maximize the rate. In this context, it is shown that the inclusion of the discrete bitloading constraint in the optimization problem may lead to game with multiple Nash equilibria or even with no equilibrium, depending on the characteristics of the communication environment. Simulations in representative DSL scenarios are presented and the results of the iterative discrete bitloading are compared with those of the well known iterative water-filling algorithm.

Keywords-Digital subscriber lines, Distributed spectrum management, Muti-carrier systems.

\section{INTRODUCTION}

The digital subscriber lines (DSL) technology uses the telephone twisted copper pairs to provide high-speed data services [1]. Twisted pairs from different users are normally grouped in binders, which may contain many pairs. Due to the proximity of these pairs, their signals are electromagnetically coupled, generating crosstalk between the pairs. The crosstalk is one of the main impairments of DSL systems, and one of the main factors limiting the achievable data rates of these systems.

Spectrum management is a central issue in the design of interference-limited multiuser communication systems such

Rafael Ferrari, Renato R. Lopes and João M. T. Romano, School of Electrical and Computer Engineering, University of Campinas - UNICAMP, 13083-970, Campinas, SP, Brazil, E-mails: \{rferrari,rlopes\}@decom.fee.unicamp.br,romano@dmo.fee.unicamp.br as DSL. The DSL environment is frequency selective, so the power available to each user must be allocated over different frequencies. Also, due to the crosstalk, each user's performance depends not only on his own power allocation, but also on that of other users in the binder.

Most of the current DSL systems are designed as singleuser systems and employ a fixed transmit power spectral density (PSD) for all modems, not taking into account the loop environment [1]. This approach leads to rates well below the system multiuser capacity. However, the increasing demand for higher data rates requires better power control schemes. Several dynamic spectrum management (DSM) techniques have been developed in the recent years to allow the users to optimize their PSD's according to the DSL loop environment [2]-[5]. These techniques permit noticeable rate improvement in the DSL systems when compared to the fixed PSD approach.

The well-known DSM algorithm named iterative waterfilling (IWF) [2] is considered in this work. This algorithm is particularly interesting from a practical point of view because it requires minimal changes in the current DSL systems: the IWF is a distributed and asynchronous DSM technique that does not require coordination between the users. The IWF is based on the formulation of the multiuser environment as a noncooperative game. An important feature of the IWF is that it converges to a unique Nash equilibrium under a wide range of conditions, which are met by practical DSL systems [2]. However, the resulting bit allocations of the IWF are continuous. This is an issue for practical deployment because the modulation scheme used in the DSL systems requires discrete bit allocations.

The main objective of this work is to analyze the impact of the discrete biloading constraint in the convergence and in the performance of autonomous DSM. The analysis is based on game theory and computational simulations using DSL scenarios.

The rest of the paper is organized as follows. Section II presents the DSL channel model and reviews the IWF algorithm. Section III presents an autonomous DSM algorithm based on iterative discrete bitloading and analyzes its convergence. Simulation results comparing the performance and convergence of the iterative discrete bitloading with the IWF are presented in section IV. Conclusions are drawn in section V. 


\section{ITERATIVE WATER-FILLING}

DSL systems use discrete multi-tone (DMT) modulation to transform the time-dispersive channel into multiple parallel channels in the frequency domain, called subchannels or tones, with no intertone-interference. In a scenario with $N$ users, the channel output at a given tone $k$ is given by

$$
\mathbf{y}_{k}=\mathbf{H}_{k} \mathbf{x}_{k}+\mathbf{z}_{k}
$$

where $\mathbf{x}_{k} \triangleq\left[x_{k}^{1}, \ldots, x_{k}^{N}\right]^{\mathrm{T}}$ and $\mathbf{y}_{k} \triangleq\left[y_{k}^{1}, \ldots, y_{k}^{N}\right]^{\mathrm{T}}$ are respectively the vectors containing the signals transmitted and received of each user, $\mathbf{H}_{k}$ is a matrix that models the channel gain and crosstalk between the pairs and $\mathbf{z}_{k} \triangleq\left[z_{k}^{1}, \ldots, z_{k}^{N}\right]^{\mathrm{T}}$ represents the additive white Gaussian noise (AWGN) with covariance matrix $\sigma_{k}^{n} \mathbf{I}_{N}$.

A key problem in DSL systems is the spectrum management of the users, i.e., the design of the PSD of each user to achieve a certain quality of service. A well-known algorithm for autonomous spectrum management is the iterative waterfilling, shown in Fig. 1. In this algorithm, each user, one at a turn, optimizes his PSD in order to maximize his rate at a given probability of error and noise margin, solving the rate maximization problem

$$
\begin{gathered}
\mathbf{s}_{\mathrm{opt}}^{n}=\underset{\mathbf{s}^{n}}{\arg \max } \sum_{k} b_{k}^{n}, \\
\text { s.t. } \sum_{k} s_{k}^{n} \leq P_{n} \\
s_{k}^{n} \geq 0, \forall k
\end{gathered}
$$

where $s_{k}^{n}=\mathrm{E}\left\{\left|x_{k}^{n}\right|^{2}\right\}$ is the transmit power of the $n$-th user at tone $k, \mathbf{s}^{n}=\left[s_{1}^{n}, \ldots, s_{K}^{n}\right]^{\mathrm{T}}$ is the vector containing the power allocation of user $n, P_{n}$ is the power budget of user $n$ and $b_{k}^{n}$ is the number of allocated bits in the $k$-th tone of user $n$, computed as

$$
b_{k}^{n}=\log _{2}\left(1+\frac{1}{\Gamma} \operatorname{SINR}_{k}^{n}\right),
$$

where $\Gamma$ is the signal to noise ratio (SNR) gap to the theoretical capacity [6], which is calculated based on the target probability of error, on the coding gain and on the system noise margin, and the $\operatorname{SINR}_{k}^{n}$ is the signal to interference-plus-noise ratio (SINR) of the $n$-th user at tone $k$ and is defined as

$$
\operatorname{SINR}_{k}^{n}=\frac{\left|h_{k}^{n, n}\right|^{2} s_{k}^{n}}{\sum_{m \neq n}\left|h_{k}^{n, m}\right|^{2} s_{k}^{m}+\sigma_{k}^{n}},
$$

where $h_{k}^{n, m}=\left\{\mathbf{H}_{k}\right\}^{n, m}$. The rate maximization is a convex optimization problem whose solution is obtained through the water-filling algorithm [7].

The IWF algorithm can be divided in two stages, the inner and outer loops. The inner loop of IWF, corresponding to lines 3 to 8 in Fig. 1, represents the competition between the users. Each user, one at a turn, unilaterally determines the transmit PSD that maximizes his rate, $R_{n}$, using the water-filling algorithm subject to a power restriction $P_{n}$ and treating the interference from the others users as noise. This competition can be interpreted as a nonzero sum and noncooperative game where the users are the players and the action is the PSD

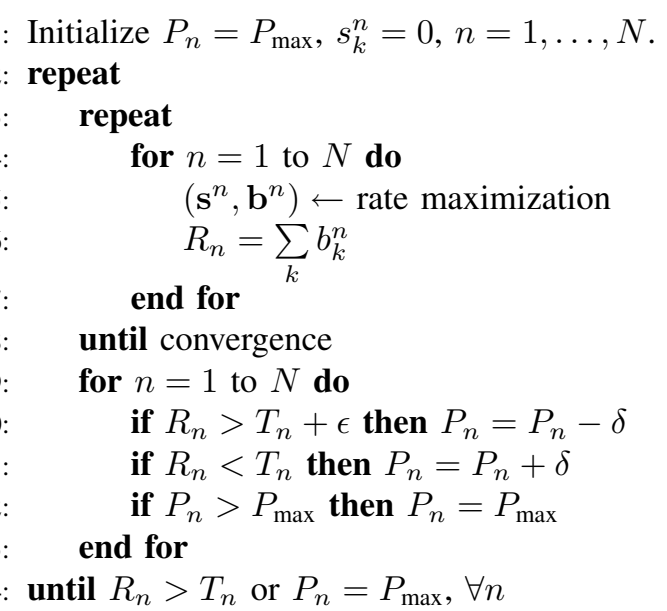

Fig. 1. Iterative water-filling algorithm.

optimization [2], [8]. In this context, the concept of Nash equilibrium can be used to determine the convergence of the competition: the competition stops when a Nash equilibrium is reached.

The outer loop of the algorithm adjusts the total power of each user taking into account the result of the iterative waterfilling and the users' target rates, $T_{n}, n=1, \ldots, N$. If the rate of a user exceeds his target by more than a given level $\epsilon$, his total power is decreased by $\delta$. Otherwise, if the rate is below the desired target, the total power is increased by $\delta$, as long as this does not exceed the maximum power value $P_{\max }$. The outer loop stops when the set of target rates is achieved or the maximum power is used. Therefore, the outer loop adjusts the total power constraint of the users so they use the minimum necessary power to achieve their target rate.

The outer loop does not influence the convergence analysis of the algorithm. The convergence of the IWF depends on the existence of a Nash equilibrium point in the noncooperative game played by the users in the inner loop. It has been shown that the IWF converges to a unique Nash equilibrium from any starting point if some conditions related to the system parameters are satisfied [2]. Fortunately, these conditions are usually met in practical systems. The IWF solutions are not globally optimal in general because the global optimum is usually attained only with coordination between the users, which is not possible in a distributed approach. However, at the Nash equilibrium, they are considered optimal in a competitive sense since the PSD of each user corresponds to the optimal solution of the rate maximization problem from a single user point of view [2].

\section{ITERATIVE DISCRETE BITLOADING}

In practical systems, there is an additional aspect that should be observed in the rate maximization problem: the number of allocated bits in a subchannel must be integer or a multiple of some base value. To simplify the analysis but without loss of generality, consider that $b_{k}^{n}$ should be integer. The bit allocation resulting from the IWF is not integer in general 
and something must be done to match the integer bitloading constraint required for practical applications. A simple solution is to maintain the power allocation unchanged and discretize the bit allocation, rounding down the number of allocated bits in each subchannel to the nearest integer. The IWF with discretized bit allocation will be called hereinafter discretized iterative water-filling (DIWF). Note that since the number of bits in each tone after the rounding operation is less than or equal to the original value, the rate of the DIWF is always less than or equal to the rate of the IWF, with equality only when the bit allocation obtained using the IWF is already integer. Also, since the PSD remains unchanged, the noise margin of DIWF in each subchannel is greater than or equal to the margin of the IWF.

Although simple, the discretization of the bit allocation does not constitute the best solution to the problem. To attain the optimal bit allocation, each user must choose his transmit PSD and bitloading solving a new rate maximization problem that includes the integer constraint

$$
b_{k}^{n} \in\{0,1, \ldots, \bar{b}\}
$$

where $\bar{b}$ is the maximum number of bits that can be assigned to a tone. The new optimization problem is an integer programming problem which can be optimally solved using greedy discrete bitloading algorithms [3], [9]. Therefore, instead of using the water-filling algorithm in line 5 of Fig. 1 , the users must determine their transmit PSD's and bit allocations using a discrete bitloading algorithm. This new distributed DSM algorithm will be hereinafter referred as iterative discrete bitloading (IDB).

The use of a discrete bitloading algorithm to determine the transmit PSD and bit allocation of the users during the competitive phase often changes the algorithm convergence and dynamics. To verify the impact of the discrete bitloading constraint, a game theory tool named reaction curve will be used to analyze the convergence of the new algorithm. The reaction curve of a user is defined as the set of optimal PSD's of the user with respect to any possible power allocation of the other users [8]. A Nash equilibrium exists if there is a point in which the reaction curves of all users intersect [8].

Consider the simplest possible scenario: two users and a single tone. In this case, the channel is defined by a single $2 \times 2$ matrix and there are only two decision variables, the transmit power of user $1, s_{1}$, and the transmit power of user $2, s_{2}$. This simple scenario allows the graphical visualization of the reaction curves, which makes the convergence analysis easier.

Fig. 2 shows the reaction curves of a two-user scenario in which the parameters were chosen so that the convergence conditions of the IWF are respected. The reaction curve of a user is obtained by evaluating his optimal transmit power for all possible values of transmit power of the other user. It can be seen that the reaction curves are not continuous, a consequence of the integer nature of the bit allocation. Each line segment is associated to a constant number of allocated bits and there is a difference of one bit between two consecutive segments. Note that the users are employing the minimum necessary power to transmit with the given margin. Increasing the power of one user would increase his margin and the interference on the other user, but not his rate. The two intersection points between the reaction curves seen in Fig. 2 correspond to two Nash equilibria. In consequence, the convergence of the IDB depends on the the initial values of the transmit powers of the users and the uniqueness of the IWF is not valid for the IDB.

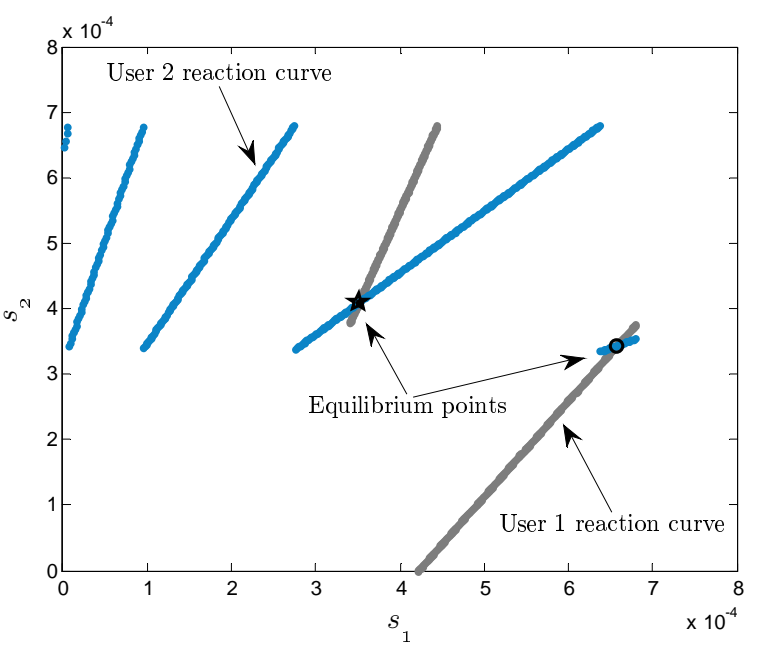

Fig. 2. A scenario with two equilibrium points.

Another scenario is depicted in Fig. 3. In this case there are no intersections between the reaction curves of the users, which means that there is no Nash equilibrium. Actually, depending on the initial power of the users, the algorithm can converge to one of the two limit-cycles shown in the figure.

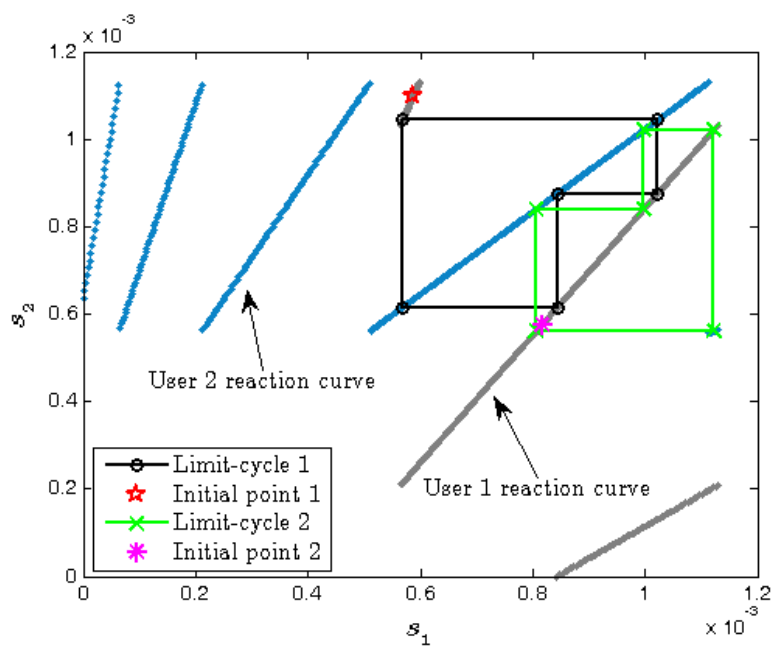

Fig. 3. A scenario with two limit-cycles.

From these two simple examples, it can be seen that the inclusion of an integer constraint on the number of allocated bits has a huge impact on the algorithm convergence. It is expected that the same impact should be observed for more complex scenarios, with more users and subchannels, like DSL systems.

Fig. 4 confirms this expectation. It depicts the rate achieved by the IWF and by the IDB algorithms in the upstream of 
a VDSL system with two users. In this figure, only one user optimizes his PSD in each iteration. The number of allocated bits in each tone and, consequently, the rate of the remaining user is simply recomputed to take into account the new interference from the optimized PSD. In order to respect the required noise margin, the number of bits in each tone is recomputed using the formula

$$
b_{k}^{n}=\left\lfloor\log _{2}\left(1+\frac{1}{\Gamma} \operatorname{SINR}_{k}^{n}\right)\right\rfloor,
$$

where $\lfloor a\rfloor$ is the largest integer not greater than $a$. The floor operation is necessary because the new SINR may lead to a non-integer allocation.

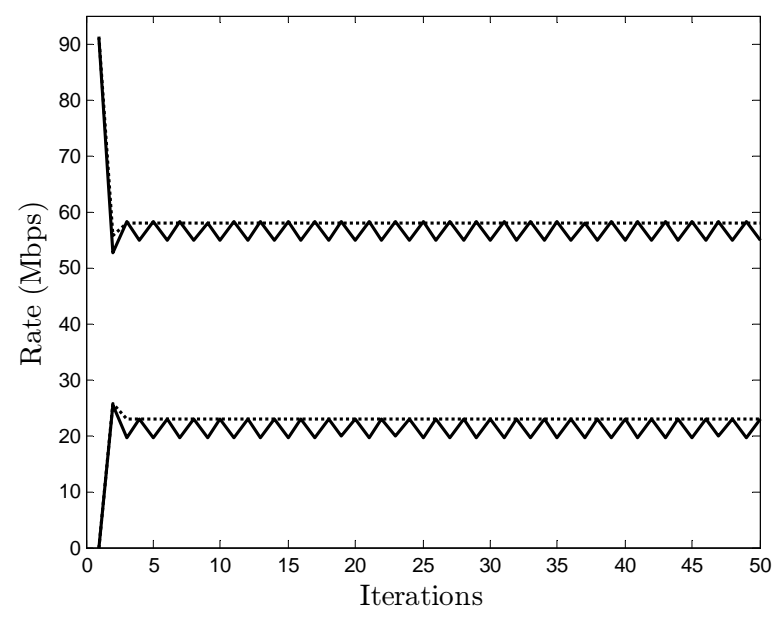

Fig. 4. Convergence of IWF (dotted lines) and IDB (solid lines) in the upstream of a VDSL system with two users. The distance between the users and the central office are $550 \mathrm{~m}$ and $700 \mathrm{~m}$.

The IWF converges to a Nash equilibrium after fewer than five iterations. However, the same equilibrium point can not be reached by the IDB due to the integer bit constraint. When there is no Nash equilibrium point, whenever a user optimizes his transmit PSD, the interference on the others users lines changes and it causes a margin loss in some of the subchannels. To maintain the margin at the required level, the number of allocated bits must be decreased on the tones where the interference has increased and, consequently, the overall rate decreases. This can be observed in Fig. 4 as, after user one optimizes his PSD, the rate of user two is updated so that the margin requirement is observed on all subchannels, leading to a rate drop. In the next iteration, user two optimizes his PSD, increasing the interference in user one, forcing him to decrease his rate to maintain the required margin. Therefore, when there is no equilibrium, only one user is operating with optimal transmit PSD and bit allocation in each iteration, while the others are operating with suboptimal power and bit allocations.

In the next section, computer simulations are performed to evaluate and compare the performance and convergence of IDB, IWF and DIWF in VDSL systems.

\section{Simulations Results}

All the simulations were performed considering the upstream channel of a VDSL system. The direct channel was generated using Chen's model [10], while the crosstalk channel was computed based on the ETSI standard [11]. The wire diameter is $0.5 \mathrm{~mm}$ (24-AWG). The target bit error rate (BER) was set to $10^{-7}$ or less $(9.8 \mathrm{~dB})$, the coding gain was set to $3.8 \mathrm{~dB}$ and the noise margin to $6 \mathrm{~dB}$, which leads to an effective gap of $\Gamma=9.8-3.8+6=12 \mathrm{~dB}$. In accordance with the VDSL standards [11], [12], the tone spacing was set to $4.3125 \mathrm{kHz}$ and the DMT symbol rate to $4 \mathrm{kHz}$. Background noise is composed of white thermal noise with a PSD of $-140 \mathrm{dBm} / \mathrm{Hz}$ and the available transmit power for each line is $11.5 \mathrm{dBm}$. Moreover, the bandplan $\mathrm{A}$ is used [13]. The maximum number of bits allowed in each tone was set to 15 and, to make comparison fair, this constraint was also applied to IWF.

In the first simulation, a scenario with two users is considered. The first user is at a distance of $400 \mathrm{~m}$ from the central office (CO), operating at a fixed rate of 50Mbps. For the simulations using the IDB, this user was chosen to be the last to update his PSD and, consequently, his bit and power allocations are optimal. A second user is placed at different distances from the CO, varying from $500 \mathrm{~m}$ to $1200 \mathrm{~m}$ in $100 \mathrm{~m}$ increments. The rate of the second user is maximized for each distance using the IWF and the IDB. The results are shown in Fig. 5.

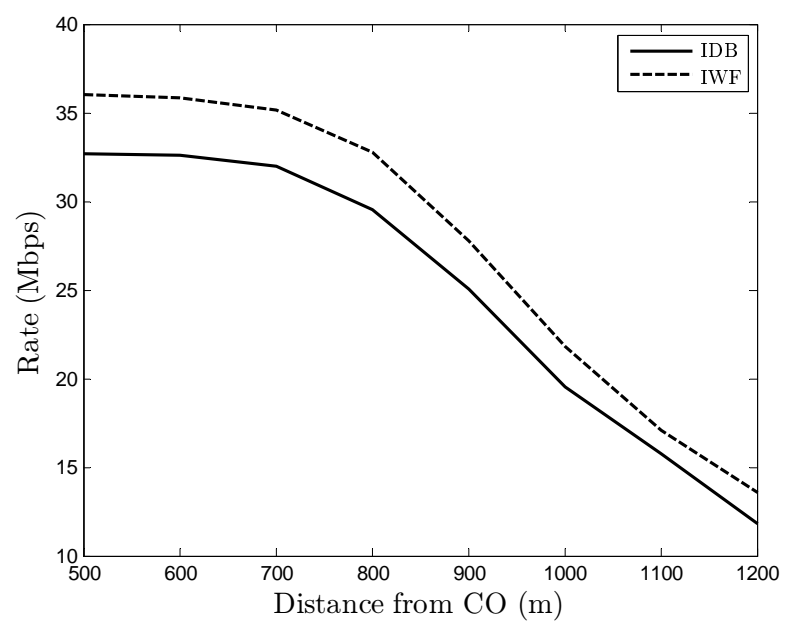

Fig. 5. Rate as a function of the reach for IWF and IDB in the upstream of a VDSL system.

For the IDB, there was no equilibrium for any of the distances, and the bit allocation in each subchannel of the second user was adjusted to maintain the noise margin equal to or higher than the specified $6 \mathrm{~dB}$. The suboptimal bit allocation of the IDB leads to a rate loss of about $10 \%$ for all distances when compared to IWF.

The next simulation compares the rate regions of IDB, IWF and DIWF. The simulation scenario is composed of two users placed respectively $400 \mathrm{~m}$ and $700 \mathrm{~m}$ far from the $\mathrm{CO}$. The results are shown in Fig. 6. In no trial using the IDB an equilibrium point was attained, indicating that the existence of a Nash equilibrium in practical scenarios is rare. Due to this convergence issue, the rates of IDB were measured after a fixed number of iterations, sufficient for the algorithm to reach 
its steady state. There are two curves for the IDB algorithm, in each of which one of the users is operating with optimal transmit PSD and bitloading.

Comparing the results, the difference between the two IDB rate regions is very small, with advantage to the case where the user connected to the $700 \mathrm{~m}$ line is operating with optimal allocations. The IWF rate region is slightly larger than the two IDB rate regions and the DIWF presents the worst performance. It is important to remember that, for the IDB, the bit allocation of one of the users is optimal and, therefore, only one user is suffering rate loss due to the lack of an equilibrium. On the other hand, for the DIWF, the rates of both users are being affected by the discretization of the bit allocation. Thus, it is possible that the impact of the absence of a Nash equilibrium in the performance of the IDB in a scenario with more users be more significant than the observed in Fig 6 , but such results are difficult to visualize since they can not be shown in a two-dimensional graphic.

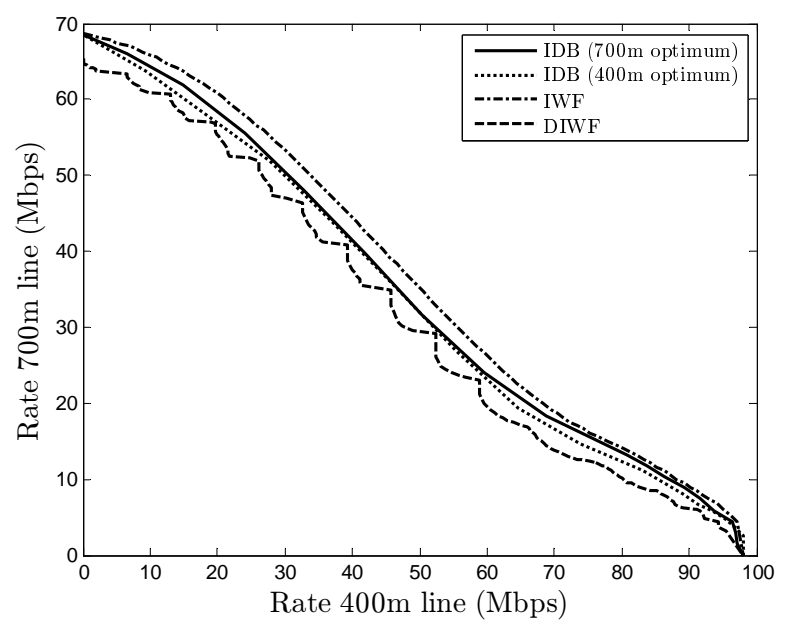

Fig. 6. Rate regions for IDB, IWF and DIWF in the upstream of a VDSL system.

The last simulation assesses the convergence and performance of the IDB in a scenario with five users. The distances between the users and the $\mathrm{CO}$ are set to $400 \mathrm{~m}$, $500 \mathrm{~m}, 600 \mathrm{~m}, 700 \mathrm{~m}$ and $800 \mathrm{~m}$. The convergence of IDB, IWF and DIWF are depicted in Fig. 7. The IDB does not have an equilibrium point and it converges to its steady state dynamic approximately in the same number of iterations that the IWF converges to its Nash equilibrium. The variation of the IDB rates are bounded and the upper bound is close to the rate achieved by the IWF while the lower bound is close to the DIWF rate. In this scenario, the maximum IDB rate loss, i.e., the difference between the maximum and the minimum rate in the steady state, varies from approximately $6 \%$ to $18 \%$, as can be seen in Table I. The relation between the upper bound and the IWF rate can be explained by the fact that the peaks of the IDB rate occur when the bit allocation is optimal and the optimal solutions of the rate maximization problem with or without the integer bitloading constraint are close. However, the relation between the minimum value of the IDB rate and the DIWF rate is not obvious and further
TABLE I

IWF, IDB AND DIWF RATE COMPARISON FOR THE SCENARIO WITH FIVE USERS

\begin{tabular}{|c||c|c|c|c|c|}
\hline \multicolumn{1}{|c||}{} & \multicolumn{5}{c|}{ Rate (Mbps) } \\
\hline Line length (m) & 500 & 600 & 700 & 800 & 900 \\
\hline \hline IWF & 49.87 & 27.25 & 16.27 & 9.97 & 6.66 \\
\hline IDB (max) & 49.78 & 27.13 & 16.18 & 9.94 & 6.68 \\
\hline IDB (min) & 46.7 & 23.96 & 13.34 & 8.17 & 5.62 \\
\hline DIWF & 47.72 & 23.95 & 13.00 & 8.07 & 5.63 \\
\hline
\end{tabular}

studies are necessary to explain this behavior.

Fig. 8 shows the sum of the rates of all users along the iterations of IWF, IDB and DIWF. After convergence, the total rate of IWF is about $9 \%$ larger than the total rate of IDB. On the other hand, the IDB outperforms the DIWF by approximately $3 \%$. The slight advantage of IDB in relation to DIWF is due to the fact that in the IDB one of the users is operating with optimal bit allocation in all iterations while the allocations of all users in the DIWF are suboptimal. Therefore, in terms of total information that can be transmitted through the channel, the use of a discrete bitloading algorithm is slightly better than the discretization of the result of the waterfilling.

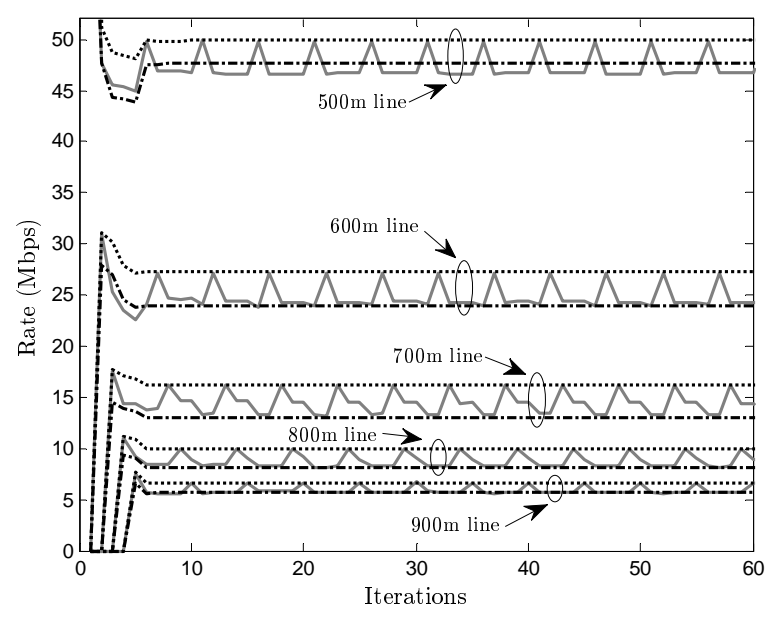

Fig. 7. Convergence of IWF (dotted lines), IDB (solid lines) and DIWF (dash dotted lines) in the upstream of a VDSL system with five users. The distances between the users and the central office are $500 \mathrm{~m}, 600 \mathrm{~m}, 700 \mathrm{~m}$, $800 \mathrm{~m}$ and $900 \mathrm{~m}$.

\section{CONCLUSION}

In this work, the convergence and performance of the IDB, an autonomous DSM algorithm based on iterative discrete bitloading, were analyzed. The motivation for this analysis is that the discrete bitloading restriction is particularly important for practical applications. Using simulation results, it was shown that the noncooperative game that represents the autonomous DSM algorithm may have multiple Nash equilibria or even no equilibrium at all when discrete bitloading algorithms are used to determine the transmit PSD's and bit allocations of the users. This result is different of that 


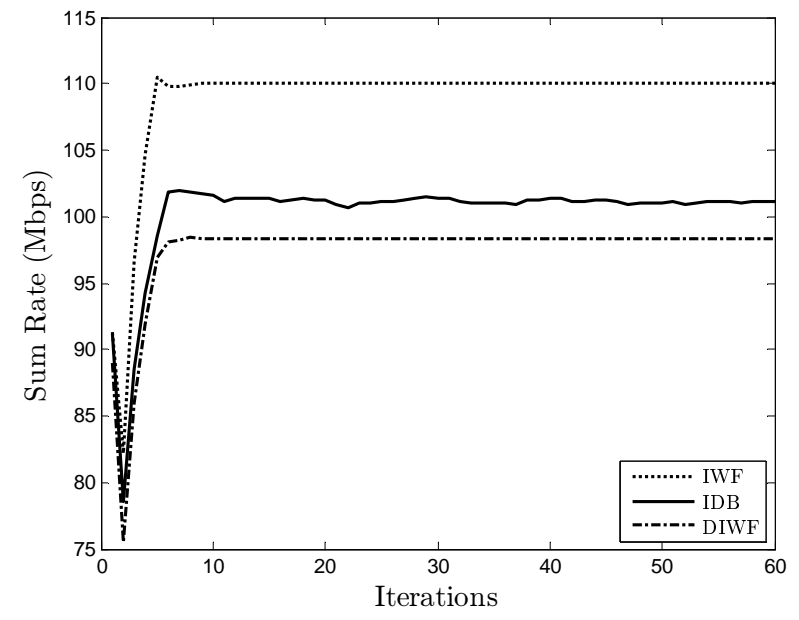

Fig. 8. Sum of the rates of all users along the iterations of IWF, IDB and DIWF for the scenario with five users.

observed in IWF which, using continuous bitloading, is proven to converge to a unique Nash equilibrium.

The results of the simulations performed in VDSL systems showed that the IDB usually does not converge to an equilibrium point. In the absence of an equilibrium, the IDB has a bounded steady state dynamic in which only the last user to maximize his rate is operating with optimal bit and power allocations. It was observed that the lower bound of the rate of the remaining users is very close to the rate achieved by the discretization of the results of IWF. However, a slight advantage is observed for the IDB in relation to DIWF if the total rate, i.e., the sum of the rates of all users, is considered. In relation to IWF, the simulations showed that the performance loss of IDB of the users operating with suboptimal allocations is approximately in the range of $5 \%$ to $20 \%$.

\section{ACKNOWLEDGMENT}

The authors would like to thank CAPES and CNPq for the financial support

\section{REFERENCES}

[1] T. Starr, J. M. Cioffi, and P. J. Silverman, Understanding Digital Subscriber Line Technology, ser. Prentice Hall Communications Engineering and Emerging Technologies Series. Prentice Hall, 1999.

[2] W. Yu, G. Ginis, and J. Cioffi, "Distributed Multiuser Power Control for Digital Subscriber Lines," IEEE J. Select. Areas Commun., vol. 20, pp. 1105-1115, Junho 2002.

[3] D. Hughes-Hartogs, "Ensemble modem structure for imperfect transmission media," US Patent 5054 034, October 1991.

[4] J. Lee, R. V. Sonalkar, and J. M. Cioffi, "Multiuser Bit Loading for Multicarrier Systems," IEEE Transactions on Communications, vol. 54, no. 7, pp. 1170-1174, July 2006.

[5] R. Cendrillon, W. Yu, M. Moonen, J. Verlinden, and T. Bostoen, "Optimal Multiuser Spectrum Balancing for Digital Subscriber Lines," IEEE Trans. on Communications, vol. 54, no. 5, pp. 922-933, Maio 2006.

[6] G. D. Forney and M. V. Eyuboglu, "Combining Equalization and Coding Using Precoding," IEEE Communications Magazine, vol. 29, no. 12, pp. 25-34, December 1991.

[7] N. Papandreou and T. Antonakopoulos, "Bit and power allocation in constrained multicarrier systems: The single-user case," EURASIP Journal on Advances in Signal Processing, vol. 2008, pp. Article ID 643 081, 14 pages, 2008, doi:10.1155/2008/643081.

[8] T. Basar and G. J. Olsder, Dynamic Noncooperative Game Theory, 2nd ed. Society for Industrial and Applied Mathematics, 1999.

[9] J. Campello, "Practical Bit Loading for DMT", in Proc. IEEE International Conference on Communications (ICC'99), Vancouver, Canada, June 1999, pp. 801-805.

[10] W. Y. Chen, DSL: Simulation Techniques and Standards Development for Digital Subscriber Line Systems. Macmillan Technical Publishing, 1998.

[11] "Very High Speed Digital Subscriber Line (VDSL); Part 1: Functional Requirements," ETSI Std. TS 101 270-1, 2003.

[12] "Very-high bit-rate Digital Subscriber Lines (VDSL) Mettalic Interface," ANSI Std. T1.424, 2004.

[13] ITU-T, G.993.1 VDSL (Very High Speed Digital Subscriber Line Transceivers), International Telecommunication Union, 2004. 